\title{
Untersuchungen zur hygienischen Qualität kleiner Fließgewässer
}

Zusammenfassung: Die Nutzung von Fließgewässern zu Erholungs- und Freizeitzwecken kann aufgrund erhöhter Konzentrationen an hygienisch relevanten Bakterienformen eingeschränkt sein. Die Quellen dieser Belastung können punktueller und diffuser Art sein. Bei Nutzung der Gewässer als Badegewässer schreibt die europäische Badegewässerrichtlinie Grenzwerte für bestimmte Indikatororganismen vor, um das Infektionsrisiko zu minimieren.

Der vorliegende Beitrag befasst sich mit den Eintragspfaden von Fäkalkeimen, und somit potentiell auch mit Krankheitserregern, in kleine Fließgewässer. Es werden Untersuchungsergebnisse von zwei Fließgewässern mit völlig unterschiedlichen Einzugsgebieten vorgestellt. Bei den Untersuchungen wurden sowohl Kläranlagenabläufe als auch Regenüberlaufbecken beprobt und auf die Keimparameter E.coli und intestinale Enterokokken nach EG-Badegewässerrichtlinie (2006/7/EWG) analysiert. Die Gewässer wurden von der Quelle an über den Fließverlauf untersucht. Im Vordergrund der Untersuchungen standen die Auswirkungen der Abwassereinleitungen auf die Fließgewässer bei Trocken- und bei Regenwetter.

Die Untersuchungsergebnisse haben deutlich gemacht, dass neben den Einleitungen von Siedlungsabwässern auch die diffusen Einträge für die hygienische Gewässerbelastung bedeutend sind. Gewässerbereiche ohne Einleitungen von Siedlungsabwässern waren bereits erheblich belastet und erreichten die Anforderungen nach EG-Badegewässerrichtlinie (2006/7/ EG) nicht. Bei Trockenwetter trugen Kläranlagen mit konventioneller Nachklärung zusätzlich zu der Keimbelastung im Gewässer bei. Kläranlagen mit weitergehenden Reinigungsstufen, wie z.B. Sandfilter, lagen hinsichtlich der Keimbelastung in der gleichen Größenordnung wie die im Gewässer gemessenen Keimkonzentratio-

\footnotetext{
Universität Stuttgart, Institut für Siedlungswasserbau, Wassergüte- und Abfallwirtschaft, Deutschland

2 SA Water Corporation, Adelaide, Australia (ehemals 1)
}

nen. Bei Regenwetter führten die Einträge aus Mischwasserentlastungen zu Spitzenkonzentrationen im Gewässer. Dennoch ist die Effizienz weitergehender Abwasserreinigungsmaßnahmen zu hinterfragen, wenn nicht gleichzeitig auch eine Reduzierung der diffusen Einträge erreicht werden kann.

Studying the hygienic quality

of small water courses

Summary: There is a risk that the use of water courses for recreation and leisure will be hampered due to major concentrations of hygienically relevant bacteria forms. The sources of such contamination may be both isolated and diffuse. Water courses used for bathing are subject to the EC Bathing Water Directive, which specifies limiting values for certain indicator organisms in order to minimise the risk of infection.

This paper discusses the input paths of faecal bacteria and therefore of pathogens to small water courses and presents results obtained from two water courses with entirely different catchments. Samples were taken both from treatment-plant outlets and storm overflow drains and analysed for the parameters E.coli and intestinal enterococci in compliance with the EC Bathing Water Directive (2006/7/EEC). The water courses were studied from their sources. The main emphasis was placed on the impact of waste-water input on the streams in both dry and wet weather.

The results of these studies have clearly shown that not only the input of residential waste water but also diffuse inputs are significant factors of hygienically relevant water pollution. Even stream sections free from residential waste water showed substantial pollution levels and did not meet the requirements set forth in the EC Bathing Water Directive (2006/7/EEC). During dry weather, sewage treatment plants equipped with conventional post-clarification facilities added to the bacterial pollution of the water course. Treatment plants with additional clarification stages, such as sand filters, exhibited bacterial concentrations of the same magnitude as those measured in the water course. Water from combined-sewage overflows entering the water course during rain storms led to peak concentration levels. Despite this there is reason to question the efficiency of increased sewage treatment, unless it is possible at the same time to reduce the diffuse inputs.

\section{Einleitung}

Gewässer wirken auf den Menschen unmittelbar und beeinflussen das Wohlbefinden und die Erholung. Die hygienische Qualität von Gewässern findet in der Öffentlichkeit im Zusammenhang mit Trinkwasser- und Badegewässernutzung hohes Interesse. Gewässergüte wird daher von BürgerInnen oft auch mit dem Ausmaß einer bakteriellen Belastung in Verbindung gebracht. Durch eine Vielzahl an anthropogenen Einflüssen, die die Gewässermorphologie, die Wasserqualität und das Abflussregime verändern, kann es zu einer Veränderung der Gewässerbiozönose und einer Einschränkung der Gewässernutzung kommen.

Durch eine intensive Messtätigkeit an den Gewässern besteht heute ein recht guter Überblick über die Belastung der Oberflächengewässer mit einzelnen, chemisch/ physikalisch leicht erfassbaren Schadstoffen, die auch im Kläranlagenablauf überwacht werden und hinreichend bekannt sind. Bei Nutzung der Gewässer als Badegewässer spielt jedoch der Gehalt an bestimmten hygienisch bedenklichen Organismen eine Rolle. In der Literatur werden für die schlechte (hygienische) Qualität der Gewässer maßgeblich die anthropogenen Belastungen, vor allem Mischwasserentlastungen, verantwortlich gemacht (Güde 2001; Overath et al. 2000; Wuhrer 1995). Diese belasten die Gewässer nicht nur hygienisch-biologisch, sondern auch optisch (Schaumbildung, Hygieneartikel etc.). Die bislang durchgeführten Untersuchungen beziehen sich allesamt auf den Bewertungsmaßstab der „alten“ EG-Badegewässerrichtlinie (76/160/EWG). Die Novellierung dieser Richtlinie (2006/7/EG) 
weist deutliche Unterschiede der zu untersuchenden Parameter, deren Grenzwerte und bezüglich der Analysemethoden auf.

Um die hygienische Gewässerqualität einschätzen und gegebenenfalls verbessern zu können, wurden an zwei kleinen Fließgewässer Monitoringprogramme hinsichtlich der siedlungswasserwirtschaftlichen Eintragspfade von Fäkalkeimen sowie deren Auswirkungen auf die Gewässer durchgeführt. Dazu wurden die Emissionen der Siedlungsabwässer sowie repräsentative Stellen im Gewässer auf die jeweilige Keimbelastung (Escherichia coli, intestinale Enterokokken) untersucht. Detaillierte Untersuchungsergebnisse finden sich in Gasse (2009).

\section{Die EG-Badegewässer- Richtlinie}

Seit über 30 Jahren wird die Qualität der Badegewässer in Europa nach der Richtlinie aus dem Jahr 1975 (76/160/EWG) anhand von fünf mikrobiologischen Parametern und 14 physikalischen bzw. chemischen Parametern überwacht. Dabei sind vor allem die mikrobiologischen Verunreinigungen einer Badestelle von Bedeutung, die unter anderem zu Durchfall und Erbrechen führen können. Für die Nutzung abwasserbelasteter Oberflächengewässer als Badegewässer gibt diese Richtlinie Leit- bzw. Grenzwerte für hygienisch relevante Mikroorganismen, wie Gesamt- und Fäkalcoliforme, Fäkalstreptokokken, Salmonellen und Darmviren an. Diese Indikatorkeime zeigen das Vorliegen von Verunreinigungen mit Warmblüterfäkalien an. Die Qualitätsanforderungen der Badegewässerrichtlinie gelten als erfüllt, wenn die Grenzwerte in $95 \%$ bzw. die Leitwerte in $80 \%$ der Proben bei gesamt- und fäkalcoliformen Bakterien und in $90 \%$ bei Fäkalstreptokokken unterschritten werden (76/160/EWG, Art. 5, Abs. 1).

Seit März 2006 ist die neue EG-Badegewässerrichtlinie (2006/7/EG) in Kraft getreten, die die „alte“ Badegewässerrichtlinie schrittweise ablöst und mit Wirkung zum 31.12.2014 aufhebt. Die Qualität der Badegewässer wird anhand der neuen Richtlinie 2006/7/EG statt durch fünf nur noch durch zwei mikrobiologische Parameter bewertet. Die Grenzwerte bezüglich des Kriteriums E.coli wurden verschärft und mit den intestinalen Enterokokken eine bislang nicht obligatorisch berücksichtigte Keimgruppe aufgenommen. Die Darmenterokokken und E.coli sollen das Verhältnis zwischen Fäkalverschmutzung

TABELLE 1

Qualitätsanforderungen nach der neuen Badegewässerrichtlinie 2006/7/EG

\begin{tabular}{|c|c|c|c|}
\hline Mikrobiologische Parameter & $\begin{array}{l}\text { Ausgezeich- } \\
\text { nete Qualität }\end{array}$ & Gute Qualität & $\begin{array}{c}\text { Ausreichende } \\
\text { Qualität }\end{array}$ \\
\hline \multicolumn{4}{|c|}{ Binnengewässer } \\
\hline Intestinale Enterokokken in cfu/100 ml & $200\left(^{*}\right)$ & $400\left(^{*}\right)$ & $330(* \star)$ \\
\hline Escherichia coli in cfu/100 ml & $500\left(^{*}\right)$ & $1.000(*)$ & $900\left(^{(\star}\right)$ \\
\hline \multicolumn{4}{|c|}{ Küstengewässer und Übergangsgewässer } \\
\hline Intestinale Enterokokken in cfu/100 ml & $100\left(^{*}\right)$ & $200\left(^{*}\right)$ & $\left.185^{(* *}\right)$ \\
\hline Escherichia coli in cfu/100 ml & $250\left(^{\star}\right)$ & $500\left(^{\star}\right)$ & $500\left(^{(\star}\right)$ \\
\hline
\end{tabular}

und Gesundheitsrisiko in Erholungsgewässern am besten widerspiegeln. Nach Richtlinie 2006/7/EG können Badestellen in vier Kategorien eingestuft werden, ,,ausgezeichnet", "gut", "ausreichend“ und „mangelhaft“. Die Kategorie „ausreichend“ ist die Mindestqualität, die alle Mitgliedsstaaten bis Ende der Saison 2015 erreichen müssen. Die neue Richtlinie unterscheidet zukünftig Badestellen an Küsten- bzw. Binnengewässer. Die Bewertungskriterien gibt Tabelle 1 wieder.

Für die Bewertung der Badestellen führt die neue EG-Badegewässerrichtlinie (2006/7/EG) ein neues Berechnungsverfahren ein. Die Qualitätsanforderungen der neuen Badegewässerrichtlinie gelten als erfüllt, wenn die mikrobiologischen Messungen die Grenzwerte nach einer 95bzw. 90-Perzentil-Bewertung unterschreiten (2006/7/EG, Anhang I und II). Die Perzentil-Werte müssen dabei auf Grundlage der $\log 10$-Normalwahrscheinlichkeitsdichtefunktion ermittelt werden. Der neue Bewertungsmaßstab führt neben der Verschärfung der Grenzwerte dazu, dass es an Badestellen zu Beanstandungen kommen kann, die nach alter Badegewässerrichtlinie die Anforderungen erfüllen.

\section{Eintragspfade von Fäkalkeimen}

\subsection{Kläranlagen}

Durch das Einleiten von gereinigtem Abwasser in ein Gewässer wird dieses zwangsläufig kontinuierlich mit Fäkalkeimen und Schadstoffen belastet. Im Hinblick auf die Eliminationsleistung von Belebungsanlagen mit konventionellen Nachklärbecken wurde festgestellt, dass unbeeinflusst von Witterungsbedingen Indikatorkeime wie Gesamt- und Fäkalcoliforme, Fäkalstreptokokken und Salmonellen zwischen einer und drei Zehnerpotenzen verringert werden. Eine typische Größenordnung der Belastung von Nach- klärbeckenabläufen mit fäkalcoliformen Keimen liegt bei ca. $10^{4}$ bis $10^{5} / 100 \mathrm{ml}$ (Gasse et al. 2006a, Popp et al. 2004, Popp, Schertler 2003; Kistemann et al. 2001; Güde 2001; Overath et al. 2000; Wuhrer 1995; Tobias et al. 1993). Die Keimelimination kann in Abhängigkeit der Verfahrenstechnik der Kläranlage und der Betriebsbedingungen variieren. Die im Nachklärbeckenablauf verbleibende Restbelastung liegt nach Güde (2001) ein bis zwei Zehnerpotenzen über dem Bereich der in Flüssen beobachteten Werte und trägt somit maßgeblich zur Grundbelastung eines Gewässers mit pathogenen Keimen bei. Bei größeren Fließgewässern (Verhältnis Abwasser/Gewässer < $1 / 20$ ) führten bislang Kläranlagenabläufe allein nicht zu Überschreitungen der Grenzwerte nach EG-Badegewässerrichtlinie 76/160/EWG. Nach Verschärfung des Keimparameters E.coli und Aufnahme der intestinalen Enterokokken nach der neuen Richtlinie 2006/7/EG ist der Sachverhalt nun neu zu hinterfragen.

Im Ablauf von kommunalen Kläranlagen können unter normalen Betriebsbedingungen die Grenz- und Leitwerte nach EG-Badegewässerrichtlinie aus dem Jahr 1975 (76/160/EWG) sicher eingehalten werden, wenn der konventionellen Nachklärung weitergehende Verfahren wie z. B. Sandfiltration in Kombination mit einer UV-Bestrahlung oder eine Membranfiltration nachgeschaltet werden. Durch Nachrüstung der Kläranlage mit einer Sandfiltration ist eine Reduzierung der Keimbelastung um eine Zehnerpotenz möglich (Overath et al. 2000; Wuhrer 1995). Die mittlere Eliminationsleistung einer UV-Bestrahlungsanlage liegt bei drei bis vier Zehnerpotenzen (Gasse et al. 2006a; Overath et al. 2000; Tobias et al. 1993). Auf der Kläranlage Bad Tölz wurden mittels Sandfiltration und UV-Bestrahlung gesamt- und fäkalcoliforme Keime um vier bis fünf Zehnerpotenzen reduziert und 
damit die Grenz- und Leitwerte der Badegewässerrichtlinie (76/160/EWG) sicher eingehalten (Popp et al. 2004). Mittels Membranfiltration können die Eliminationsleistungen um drei bis sechs Zehnerpotenzen (Overath et al. 2000), mittels Ozon um zwei bis drei Zehnerpotenzen (Scherb, Bauer 1978) gesteigert werden.

\subsection{Mischwasserentlastungen}

Bei starkem oder anhaltendem Regen werden die Gewässer zusätzlich zu den Abwassereinleitungen aus den Kläranlagen mit Abwasser aus den Kanalisationen belastet. Das Regenwasser aus Trennsystemen spielt gegenüber dem Abwasser aus Mischsystemen für die hygienische Belastung eines Gewässers eine geringere Rolle. Bei der Entwässerung mittels Mischkanalisation gelangt ungereinigtes bzw. mechanisch gereinigtes Abwasser aus den Regenüberläufen und Regenüberlaufbecken in das Gewässer. Problematisch sind dabei die über kurze Zeiträume eingetragenen hohen Schadstofffrachten sowie die stoßartige Abflusserhöhung.

Angaben über die Verschmutzung von Mischwasserentlastungen hinsichtlich Fäkalkeimen gibt es in der Literatur nur wenig. Für Regenüberlaufbecken wird eine Keimreduktion von maximal 0,5 Zehnerpotenzen angegeben (Overath et al. 2000). Dabei liegen die Belastungen im Mischwasser mit Fäkalcoliformen in einem Bereich von $10^{6}$ bis $10^{8} / 100 \mathrm{ml}$, die Anzahl der bislang durchgeführten Beprobungen sind jedoch nicht repräsentativ (Kistenmann et al. 2001; Overath et al. 2000; Wuhrer 1995; Krauth 1971).

Um die Keimemissionen aus Mischwasserentlastungen zu reduzieren, kann den Regenüberlaufbecken z. B. ein Retentionsbodenfilter nachgeschaltet werden. In schilfbewachsenen Retentionsbodenfiltern sind Eliminationsleistungen für Fäkalbakterien von ein bis drei Zehnerpotenzen erzielt worden (Güde 2006; Merkel et al. 2005; Overath et al. 2000). Die Leistungsfähigkeit dieses Reinigungssystems zeigte sich in den erzielten $\mathrm{Ab}$ laufkonzentrationen, die niedriger lagen als bei untersuchten Nachklärbeckenabläufen. Zusätzlich ist eine integrierte Betrachtung von Kanalnetz, Kläranlage und Fließgewässer denkbar. Auf Basis solcher Betrachtungen können sich besonders unter Regenwetterbedingungen z. B. durch höhere hydraulische Belastung der Kläranlagen zu Gunsten der Regenwasserbehandlung Optimierungspotentiale ergeben (Krebs 2002).

\subsection{Landwirtschaftliche Einflüsse}

Neben den punktuellen Belastungsquellen der Siedlungsbereiche dürfen diffuse Belastungen aus der Landwirtschaft für die Einschätzung der Gesamtbelastung nicht vernachlässigt werden. Naturgemäß sind sie jedoch ungleich schwerer zu erfassen als Belastungen aus Kläranlagen und Mischwasserentlastungen. Eine quantitative Bewertung der landwirtschaftlichen Einflüsse wird in Zukunft umso notwendiger, je mehr Kosten für den Einsatz neuer Techniken für die weitergehende Abwasserreinigung in Kläranlagen und der Mischwasserbehandlung entstehen.

Bei den diffusen Belastungen spielen vor allem Oberflächenabschwemmungen und Dränleitungen aus landwirtschaftlich genutzten Flächen, besonders nach Ausbringung von Dünger (Mist, Gülle), eine Rolle. Die Dränung verkürzt den Fließweg des Sickerwassers zum Gewässer, wodurch die Flächen früher abtrocknen und befahren werden können. Zu Oberflächenabschwemmungen kann es z.B. während sommerlicher Starkregen kommen, wenn die Regenintensität die Infiltrationsrate des Bodens überschreitet. Auch Abschwemmungen von Hanglagen sowie wassergesättigten bzw. gefrorenen Böden tragen in hohem Maße zur diffusen Belastung der Gewässer mit Fäkalkeimen, Nährstoffen und Pflanzenschutzmitteln bei. Die Höhe des Stoffaustrags über den Oberflächenabfluss hängt stark von der Höhe des Bodenabtrags, dem Zeitpunkt der Düngung und vom Niederschlagsgeschehen ab.

Beregnungsversuche auf Äckern und Wiesen und damit verbundene Untersuchungen hinsichtlich des Austrags von Fäkalkeimen sind in den letzten Jahren zunehmend durchgeführt worden (Weiss und Popp 2004; Weiss 2003). Beregnungsversuche auf gedüngten Flächen (Wirtschaftsdünger mit $10^{9}-10^{13} / 100 \mathrm{ml}$ Fäkalcoliformen und Fäkalstreptokokken) zeigten, dass die Konzentrationen im Dränaustrag zwischen $10^{7}$ und $10^{11}$ fäkalcoliformen Keimen/100 ml und zwischen $10^{8}$ und $10^{10}$ fäkalstreptokokken Keimen/100 ml liegen. Die Ausbringung von Gülle 24 Stunden vor der Bodenbearbeitung und Beregnung führte zu keinem Rückgang der Wiederfindungsrate. Das Ausschwemmungspotential von Fäkalbakterien aus Festmist lag in der gleichen Größenordnung wie bei Gülle. Gegenüber der Grundbelastung erhöhte sich die Fracht an ausgeschwemmten fäkalkoliformen Keimen um drei bis vier Zehnerpotenzen, die der Fäkalstreptokokken nur um maximal zwei Zehnerpotenzen. Bei weiterer Beregnung gingen die Frachten an Fäkalcoliformen deutlich zurück, die der Fäkalstreptokokken jedoch nicht. Das macht deutlich, dass auch wiederholter Starkregen nach einer Düngung zu einer erneuten Ausschwemmung von Fäkalbakterien führen kann.

Die Menge an ausgeschwemmten Fäkalbakterien erwies sich als unabhängig vom Standort (Wiese, Acker), der Art der Bodenbearbeitung, dem Bewuchs und der Jahreszeit. In Anbetracht dessen, dass in Deutschland ca. 2 Millionen Hektar landwirtschaftliche Nutzfläche gedränt sind (Lennartz et al. 1997), wird das Gefährdungspotential für die Hygiene der Gewässer deutlich.

Güde (2001) zeigte, dass sich die Mikroflora der Gülle mit der Lagerung ändert, wobei eine starke Abnahme der ursprünglich dominierenden Fäkalkeime beobachtet werden konnte. Nach ca. drei Monaten Lagerungszeit waren keine E.coli und kaum noch Streptokokken nachweisbar. Auch Weiss (2003) stellte einen kontinuierlichen Rückgang der fäkalcoliformen Keime um drei Zehnerpotenzen über einen Lagerungszeitaum von sechs Monaten fest.

\section{Gewässermonitoring an Stockacher Aach und Körsch}

Um die unterschiedlichen anthropogenen und natürlichen Belastungsquellen abschätzen zu können, erfolgte ein Gewässermonitoring an zwei kleinen Fließgewässern mit stark unterschiedlichem Einzugsgebiet in Abhängigkeit von Trocken- und Regenwetter.

Die Stockacher Aach (Bodenseekreis) entspringt als Mindersdorfer Aach in einem Waldgebiet südlich der europäischen Hauptwasserscheide. In direkter Nähe des Quellgebiets liegt auch der Oberlauf der Ablach, die in Richtung Norden in die Donau fließt. Im flachen Gebiet der Wasserscheide Rhein/Donau hat die Mindersdorfer Aach eine Bifurkation gebildet. Sie verzweigt sich im Gebiet der Schwackenreuter Seenplatte und fließt als Ablach in die Donau, bei erhöhter Wasserführung als Stockacher Aach in den Bodensee. Im Bereich der Mindersdorfer Aach leitet die Kläranlage Mindersdorf (Scheibentauchkörperanlage, $400 \mathrm{EW}$ ) ein, in die Stockacher Aach die Kläranlage Mühlingen (1.800 EW). Letztere stellt bei Trockenwetter die erste Siedlungsabwassereinleitung dar. Das gesamte Einzugsgebiet der Stockacher Aach ist überwiegend land- 
wirtschaftlich geprägt mit kleinen Ortschaften und Streusiedlungen. Den einzigen Ballungsraum stellt die Stadt Stockach mit 16.000 EW dar. Auf 25 Kilometern Fließstrecke leiten direkt in die Stockacher Aach 14 Regenüberlaufbecken ein, von denen zwei hinsichtlich Entlastungstätigkeit und Entlastungsfrachten genauer untersucht wurden.

Die Körsch (Gemarkung Stuttgart) entspringt ebenfalls in einem kleinen Waldgebiet, durchfließt aber auf den ersten Kilometern bereits dicht besiedelte Stadtgebiete. Das Einzugsgebiet ist stark urban geprägt, weist jedoch auch landwirtschaftlich genutzte Flächen und unberührte Gewässerstrecken (Naturschutzgebiete) auf. Die obere Körsch (Gemarkung Stuttgart) führt als relativ kleiner Vorfluter eine verhältnismäßig große Menge von geklärtem Abwasser aus den zwei Stuttgarter Kläranlagen Möhringen und Plieningen ab. Bei Niedrigwasserabfluss besteht der Bach nahezu vollständig aus gereinigtem Abwasser. Bei Starkregen wird die Körsch über die entsprechenden Mischwasserentlastungsbauwerke belastet. Allein im Stuttgarter Stadtgebiet befinden sich 23 Regenüberlaufbecken (SES 2002).
Das Monitoringprogramm an den beiden Fließgewässern gliederte sich in Trocken- und Regenwetteruntersuchungen. Durch gezielte Untersuchungen am Fließgewässer bei Regenwetter sollte vor allem der Einfluss von vermehrten Einleitungen von Siedlungsabwasser (Mischwasserentlastungen, Kläranlagenabläufe) auf das Gewässer sichtbar gemacht werden. In Abhängigkeit der möglichen landwirtschaftlichen und der siedlungswasserwirtschaftlichen Einflüsse wurde die Stockacher Aach über die gesamte, die Körsch über die obere Gewässerfließstrecke beprobt.

An der Stockacher Aach wurden neun Gewässerstellen und zwei Zuflüsse beprobt. Als Punktquellen wurde eine Kläranlage sowie zwei Regenüberlaufbecken untersucht. Von diesen Becken wurde auch die Entlastungstätigkeit und die entlastete Mischwassermenge im untersuchten Zeitraum aufgezeichnet.

An der Körsch (Oberlauf Sindelbach) wurden elf Probennahmestellen sowie zwei Zuflüsse untersucht. Zusätzlich wurden die Abläufe zweier Kläranlagen sowie bei Regenwetter die Entlastungen zweier
Regenüberlaufbecken analysiert. Entlastungshäufigkeit und Entlastungsmengen aller im Einzugsgebiet befindlichen Regenüberlaufbauwerke wurden ermittelt.

Der Untersuchungszeitraum erstreckte sich jeweils über zwei Sommerhalbjahre. Insgesamt wurden 36 Trockenwetterbeprobungen und 20 Regenwetterbeprobungen durchgeführt.

\section{Trockenwetteruntersuchungen}

Die Konzentrationen der untersuchten Kläranlagenabläufe Mühlingen (Stockacher Aach) und Möhringen (Körsch) lagen für E.coli und intestinalen Enterokokken zwischen $10^{3} / 100 \mathrm{ml}$ und $10^{5} / 100 \mathrm{ml}$ und entsprachen damit den Literaturwerten für Abläufe konventioneller Nachklärbecken (Mediane E.coli: $2,0 \cdot 10^{4} / 100 \mathrm{ml}$ und $2,5 \cdot 10^{4} / 100 \mathrm{ml}$; Mediane intestinale Enterokokken: $1,1 \cdot 10^{4} / 100 \mathrm{ml}$ und $2,3 \cdot 10^{3} / 100 \mathrm{ml}$ ). Sie lagen jeweils ein bis zwei Zehnerpotenzen über dem Belastungsniveau der Gewässer vor der Kläranlageneinleitung. Die untersuchte Kläranlage Plieningen, die über einen nachgeschalteten Sandfilter
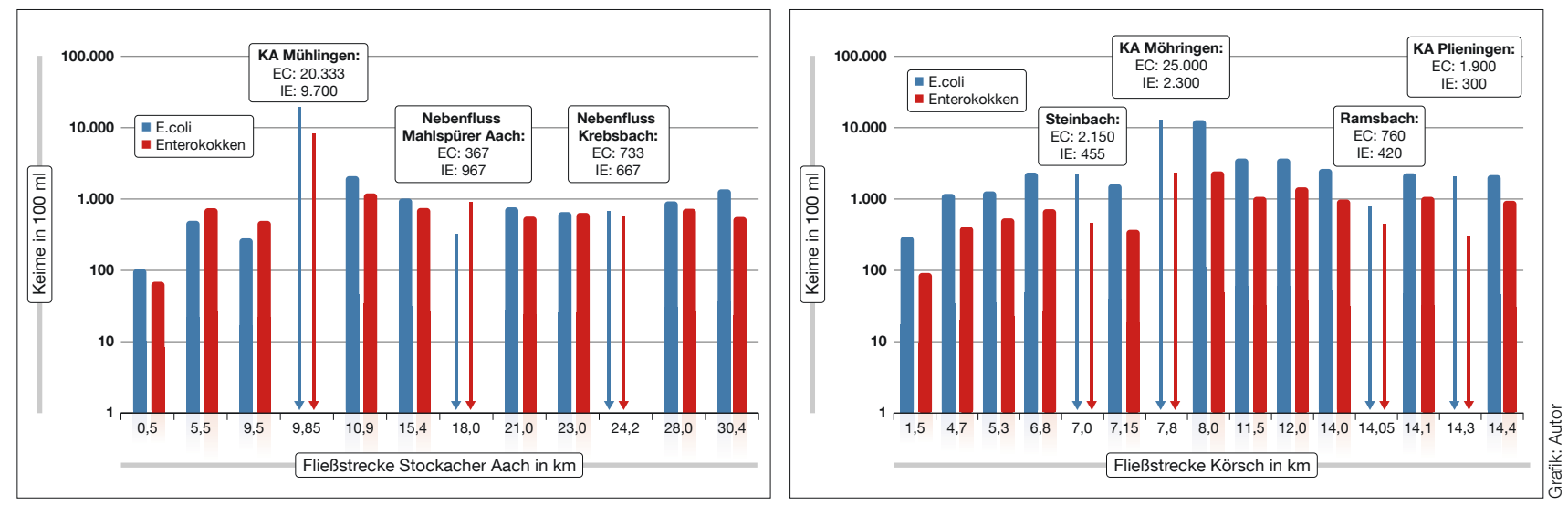

Abb. 1: Keimbelastung der Stockacher Aach und der Körsch, Medianwerte für E.coli und intestinale Enterokokken einschließlich der Nebenflüsse und der Kläranlageneinleitung bei Trockenwetter.
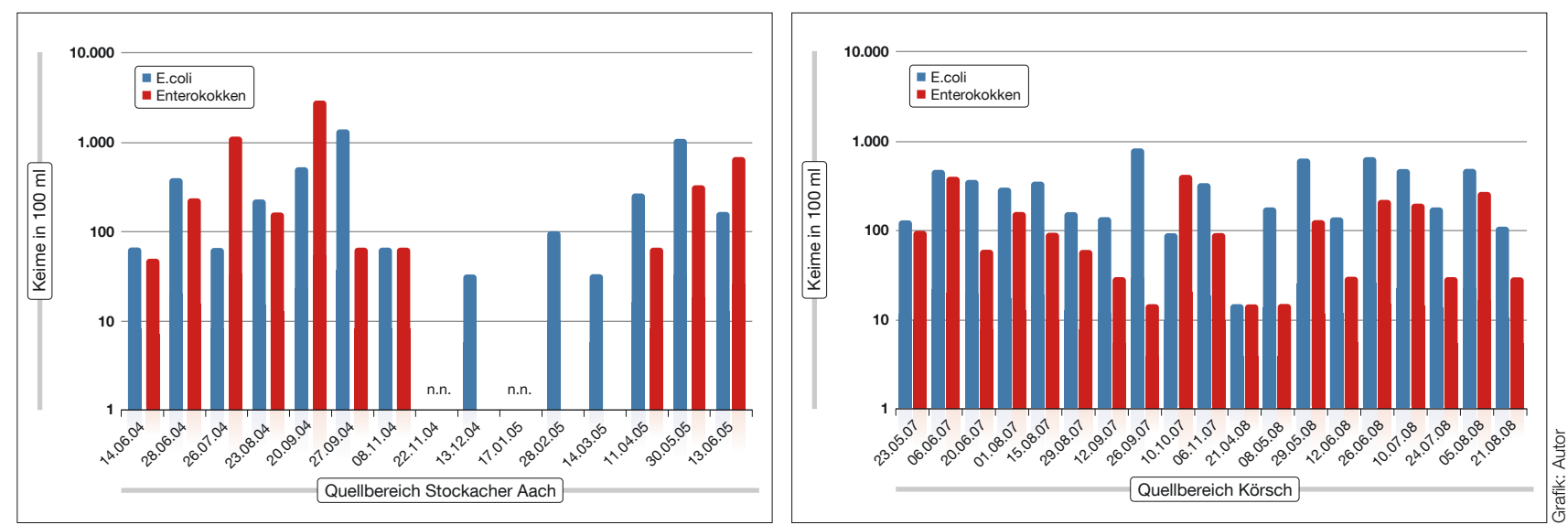

Abb. 2: Keimbelastung der Stockacher Aach und der Körsch nahe der Quelle bei Trockenwetter. 
verfügt, wies erwartungsgemäß etwa eine Zehnerpotenz geringere Keimkonzentrationen auf (Median E.coli: 3,0·102/100 ml; Median intestinale Enterokokken: $1,9 \cdot 10^{3} / 100 \mathrm{ml}$ ). Die Kläranlagenabläufe wiesen für E.coli etwa eine Zehnerpotenz höhere Konzentrationen auf als für die intestinalen Enterokokken.

Die Beprobung der Gewässer an charakteristischen Gewässerstellen ergab das in Abbildung 1 dargestellte Bild. Wider Erwarten ergab sich an beiden Gewässern bereits eine auffällig hohe hygienische Belastung im Quellbereich (siehe Abbildung 2). Die Stockacher Aach wurde in unmittelbarer Nähe der Quellschüttung mitten im Wald, die Körsch ca. 1,5 km unterhalb der Quelle nach Austritt aus einem Waldgebiet in einem Wiesenbereich beprobt.

An der Stockacher Aach lagen die Konzentrationen an der Quelle in den Wintermonaten (November bis März), als längere Frostperioden herrschten und insgesamt wenig Niederschlag fiel, deutlich niedriger als in den übrigen Monaten. Intestinale Enterokokken waren zu der Zeit an der Quelle gar nicht nachweisbar, E.coli waren zum Teil ebenfalls nicht nachweisbar, zum Teil nur bis maximal $100 \mathrm{KBE} / 100 \mathrm{ml}$. Es wird vermutet, dass die Keimbelastung der Quelle nur aus tierischen Fäkalien stammen kann. Im Sommer und Herbst überraschen die wiederholt sehr hohen Belastungen der intestinalen Enterokokken, aber auch die von E.coli. Im Quellbereich der Stockacher Aach konnten die Anforderungen nach neuer EG-Badegewässerrichtlinie (2006/7/EG) für die Kategorie gut, im Quellbereich der Körsch lediglich für die Kategorie ausreichend eingehalten werden.
Im weiteren Fließverlauf stiegen die Keimkonzentrationen in den Gewässern deutlich an. An der Stockacher Aach ist der Anstieg auf die Einleitung der kleinen Kläranlage bei km 4 (die jedoch aufgrund der Bifurkation anschließend bei Trockenwetter in die Ablach abfließt) sowie auf Emissionen von landwirtschaftlichen Flächen zurückzuführen. In stark landwirtschaftlich genutzten Gebieten überwog im Gewässer die Belastung mit intestinalen Enterokokken gegenüber den E.coli. An der Körsch erfolgen im Oberlauf weder siedlungswasserwirtschaftliche Einleitungen noch wesentliche landwirtschaftliche Flächennutzungen. Das Keimniveau steigt dennoch rasch um eine Zehnerpotenz im Gewässer an. Gründe hierfür sind nicht offensichtlich und bedürfen weiterer Untersuchungen.

Nach Einleitung des ersten Kläranlagenablaufs stieg die Gewässerbelastung mit E.coli und intestinalen Enterokokken im Median um eine Zehnerpotenz an. Konnten an der Stockacher Aach noch vor der Kläranlageneinleitung die Anforderungen nach neuer EG-Badegewässerrichtlinie (2006/7/EG) für alle Kategorien eingehalten werden $(\mathrm{km} \mathrm{9,5)}$, so wurden nach Einleitung der ersten Kläranlage und an allen nachfolgenden Gewässerstellen alle Grenzwerte deutlich überschritten. An der Körsch konnten bereits ab Messstelle 2 die Anforderungen für alle Qualitätskategorien nicht mehr eingehalten werden.

Die Kläranlage Stuttgart-Plieningen mit nachgeschaltetem Sandfilter trug nicht zu einer Erhöhung des Keimniveaus der Körsch bei, da diese geringere Ablaufkonzentrationen an E.coli und intestinalen Enterokokken aufwies als die Körsch selbst.

In Gewässerabschnitten ohne anthropogene Einleitungen konnte in beiden Ge- wässern eine Abnahme der Keimbelastung um bis zu einer halben Zehnerpotenz beobachtet werden. Diese Reduktion wird auf die Selbstreinigungskräfte des Gewässers sowie auf Sedimentation und Fraßverluste zurückgeführt.

\section{Regenwetteruntersuchungen}

In den Mischwasserentlastungen lagen die Konzentrationen für E.coli in der Größenordnung von $10^{5}$ bis $10^{7}$ Keime $/ 100 \mathrm{ml}$, für die intestinalen Enterokokken in der Größenordnung von $10^{3}$ bis $10^{6}$ Keime/100 ml (Mediane der vier untersuchten Becken: E.coli: 5,4 $10^{5} / 100 \mathrm{ml}$, $3,6 \cdot 10^{5} / 100 \mathrm{ml}, \quad 1,2 \cdot 10^{6} / 100 \mathrm{ml}$, $3,3 \cdot 10^{6} / 100 \mathrm{ml}$, intestinale Enterokokken: $8,7 \cdot 10^{4} / 100 \mathrm{ml}, 1,6 \cdot 10^{5} / 100 \mathrm{ml}, 1,4 \cdot 10^{5} / 100$ $\left.\mathrm{ml}, 7,9 \cdot 10^{5} / 100 \mathrm{ml}\right)$. Da bislang keine umfangreichen Untersuchungen im Bereich der Mischwasserentlastungen stattgefunden haben, können diese Werte nicht direkt mit der Literatur abgeglichen werden (siehe Abschnitt 3).

Die Keimkonzentrationen der zwei untersuchten Kläranlagen mit konventioneller Nachklärung lagen bei Regenwetter für E.coli in der Größenordnung $10^{4}$ bis $10^{6} / 100 \mathrm{ml}$, für die intestinalen Enterokokken in der Größenordnung $10^{3}$ bis $10^{5} / 100 \mathrm{ml}$ (Mediane: $1,9 \cdot 10^{5} / 100 \mathrm{ml}$ und $1,3 \cdot 10^{5} / 10 \mathrm{ml}$; Median intestinale Enterokokken: $2,1 \cdot 10^{4} / 100 \mathrm{ml}$ und $2,2 \cdot 10^{4} / 10 \mathrm{ml}$ ). Im Vergleich zu den Trockenwetteranalysen des regelmäßigen Gewässermonitorings waren die Ablaufwerte bei Regenwetter um ein bis zwei Zehnerpotenzen erhöht. Dabei war die Belastung des Kläranlagenablaufs mit E.coli bei Regenwetter um ca. eine bis eineinhalb Zehnerpotenzen höher als die Belastung mit intestinalen Enterokokken. Die Kläranlage Stutt-
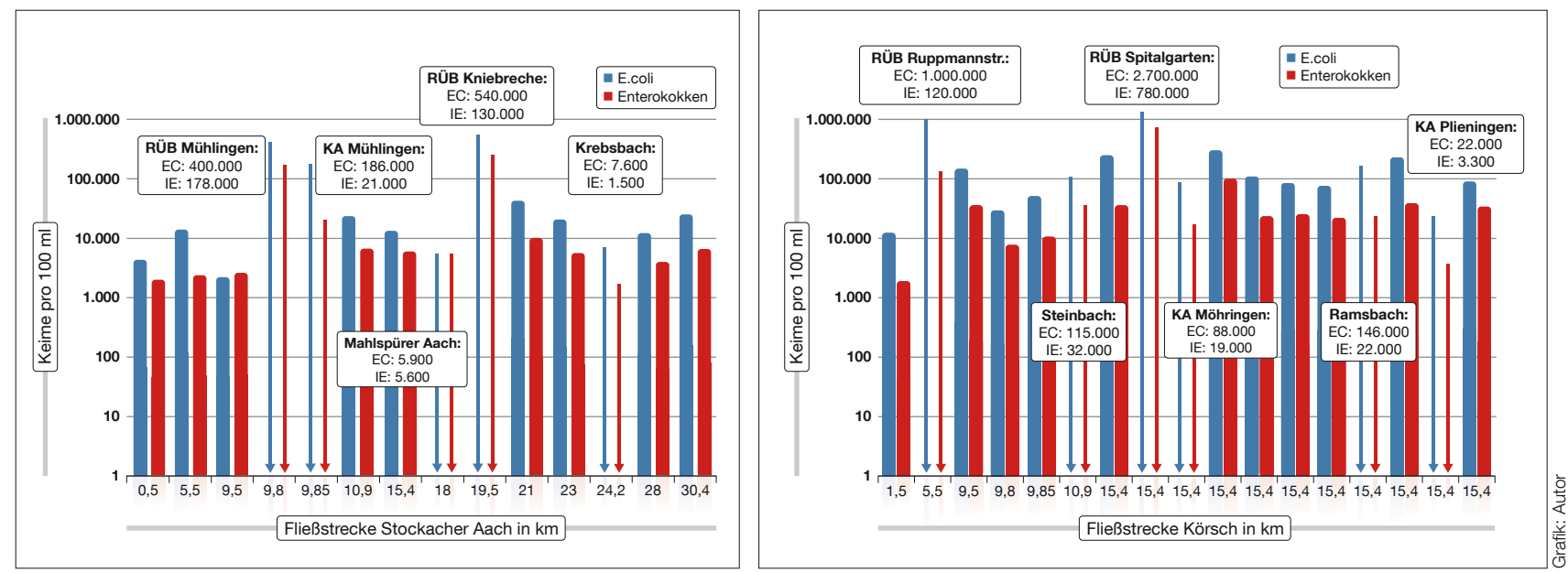

Abb. 3: Keimbelastung der Stockacher Aach und der Körsch, Medianwerte für E.coli und intestinale Enterokokken einschließlich Nebenflüsse, Kläranlagen und untersuchten Mischwassereinleitungen bei Regenwetter. 
gart-Plieningen wies durch den nachgeschalteten Sandfilter bis zu einer Zehnerpotenz geringere Keimkonzentrationen auf. Die Keimkonzentrationen im Ablauf des Sandfilters schwankten jedoch deutlich mehr als im Ablauf der konventionellen Nachklärbecken. Die Keimbelastung der beiden Gewässer bei Regenwetter stellt Abbildung 3 dar.

Die Keimkonzentrationen im Gewässer waren gegenüber denen bei Trockenwetter um ein bis zwei Zehnerpotenzen erhöht. Die Anforderungen nach EG-Badegewässerrichtlinie (2006/7/EG) konnten an keiner Stelle eingehalten werden. Daraus wird ersichtlich, dass nicht nur die Einleitungen aus der Siedlungsentwässerung eine Rolle für die hygienische Gewässerbelastung spielen, sondern vielmehr auch diffuse Einträge, natürlichen oder anthropogenen Ursprung, ausschlaggebend sein können. Die bei Regenwetter vorhandene hohe Keimbelastung im Quellbereich der Gewässer wird durch die sätzlich angehoben. Dabei können nach Einleitung von Siedlungsabwässern zu-

der Einleitung von Mischwasserentlastungen Spitzenbelastungen im Gewässer von mehr als $10^{6}$ E.coli $/ 100 \mathrm{ml}$ und über $10^{5}$ int. Enterokokken/100 $\mathrm{ml}$ nachgewiesen werden.

Gewässerabschnitte ohne anthropogene Einleitungen sind an Stockacher Aach und Körsch kaum zu finden. Dennoch konnte auf Strecken ohne punktuelle Einleitungen eine Reduktion der Keimbelastung, auch bei Regenwetter, erkannt werden. Diese sind auf Fraß- und Sedimentationsvorgänge zurückzuführen.

\section{Bewertende Zusammenfassung}

Die vorangehenden Ausführungen haben gezeigt, dass neben den Einleitungen von Siedlungsabwässern auch die diffusen Einträge für eine hygienische Gewässerbelastung eine bedeutende Rolle spielen. Das wurde besonders in den Gewässerbereichen deutlich, in denen noch keine Siedlungsabwässer eingeleitet wurden. Bei Trockenwetter waren hohe Konzentrationen an E.coli und intestinalen Entero-

\section{LITERATUR}

2006/7/EG: Richtlinie 2006/7/EG des Europäischen Parlaments und des Rates über die Qualität der Badegewässer und deren Bewirtschaftung und zur Aufhebung der Richtlinie 76/160/ EWG, ABI. EG Nr. L 64

76/160/EWG: Richtlinie 76/160/EWG des Rates über die Qualität der Badegewässer, ABI. Nr. L 3 Gasse J (2009) Quantifizierung der Emissionen aus Abwasseranlagen und deren Auswirkungen auf die hygienische Qualität von Fließgewässern, Stuttgarter Berichte zur Siedlungswasserwirtschaft, Band 198, Oldenbourg Industrieverlag München, $220 \mathrm{~S}$.

Gasse J, Schrader C, Krampe J (2006) Vergleichende Untersuchungen innovativer Verfahren zur Desinfektion der Stuttgarter Kläranlagenabläufe, Abschlussbericht im Auftrag der Stadtentwässerung Stuttgart, unveröffentlicht Güde H (2006) Persönliche Mitteilung, Institut für Seenforschung, LUBW, Langenargen Güde $\mathbf{H}$ (2001) Erfassung und Bewertung von Eintragswegen für Belastungen mit Fäkalkeimen im Einzugsgebiet der Seefelder Aach (Bodenseekreis), Abschlußbericht des BW Plus PAO 97008 Kistemann T, Koch C, Herbst S, Rechenburg A, Exner M (2001) Untersuchungen zur mikrobiellen Fließgewässerbelastung durch Kläranlagen, Abschlußbericht im Auftrag des MUNLV NRW Krauth Kh (1971) Der Abfluss und die Verschmutzung des Abwassers in Mischkanalisationen bei Regen, Stuttgarter Berichte zur Siedlungswasserwirtschaft, Band 45, Oldenbourg Industrieverlag München, $251 \mathrm{~S}$.

Krebs (2002) Integrierte Simulation von KanalisaKrebs (2002) Integrierte Simulation von Kanalisa-
tion, Kläranlage und Fließgewässer, 77 . Siedlungswasserwirtschaftliches Kolloquium, Stuttgarter Berichte zur Siedlungswasserwirtschaft, Band 169, Oldenbourg Industrieverlag München, S. 43-64 Merkel W, Grobe S, UhI M, Janiczek M, Henrichs $M$ (2005) Weitergehende Mischwasserberichs $M$ (2005) Filteraufbau und Filterbetrieb zur Verbesserung Filteraufbau und Filterbetrieb zur Verbesserung
der Reinigungsleistung insbesondere hinsichtlich hygienisch relevanter Mikroorganismen, Abschlussbericht des IWW und der Fachhochschule Münster
Overath H, Merkel W, Hiekel S (2000) Einleitungen von Kläranlagenabläufen in kleine Fließgewässer: Bewertung der Ablaufqualität nach der EG-Badegewässerrichtlinie, Abschlussbericht, IWW Rheinisch-Westfälisches Institut für Wasserforschung

Popp W, Huber S, Kexel S (2004) Abwasserdesinfektion zur Verbesserung der Badegewässerqualität an der Oberen Isar, Wasser und Abfall, Nr. 5, S. 14-18

Popp W, Schertler C (2003) Bakteriologischhygienisches Untersuchungsprogramm "Obere Isar" zur Wiederherstellung der Badegewässerqualität, Münchener Beiträge zur Abwasser-, Fischerei- und Flussbiologie, Band 55, S. 61-78

Scherb K, Bauer H (1978) Der Einsatz von Ozon zur Nachbehandlung von biologisch vorgereinigtem Abwasser, Münchner Beiträge zur Abwasser-, Fischerei- und Flußbiologie, Band 29 Abwasser-, Fischerei- und Flußbiologie, Band 29
SES (2002) Allgemeiner Entwässerungsplan der Landeshauptstadt Stuttgart, Stand: Januar 2002, Stadtentwässerung Stuttgart

Tobias H, Richter KD, Heinemeyer EA

(1993) Auswirkungen einer Abwasserreinigung durch Filtrationsverfahren und UV-Entkeimung auf die hygienische Qualität des Abwassers und auf die hygienische Qualität des Abwassers und
Basisuntersuchungen des Vorfluters Norder Tief, Bericht des Staatlichen Medizinaluntersuchungsamtes Aurich

Weiss K, Popp W (2004) Quantifizierung der diffusen Belastung von Gewässern mit Fäkalbakterien aus landwirtschaftlich genutzten Flächen,

Materialien Nr. 111, Bayerisches Landesamt für Materialien Nr. 111, Bayerisches La

Weiss K (2003) Belastung von Oberflächengewässern durch Eintrag aus landwirtschaftlich genutzten Flächen, in: Hygienische Aspekte von Oberflächengewässern aus wasserwirtschaftlicher Sicht, Münchner Beiträge zur Abwasser-, Fischerei- und Flussbiologie, Band wasser-, Fisch

55, S.79-92
Wuhrer C (1995) Die fäkale Belastung der Schussen und ihr Einfluss auf den mündungsnahen Flachwasserbereich im Bodensee, Dissertation am Institut für Seenforschung, LUBW kokken im Gewässer bereits vor der jeweils ersten Kläranlageneinleitung festzustellen, die in den meisten Fällen bereits die Anforderungen der neuen EG-Badegewässerrichtlinie (2006/7/EG) nicht erfüllten. Kläranlagen mit konventioneller Nachklärung trugen dann zusätzlich zur Keimbelastung bei. Eine Anlage mit weitergehender Reinigungsstufe (Sandfilter) führte nicht zu einer Verschlechterung der Keimkonzentrationen im Gewässer.

Bei Regenwetter lagen die Keimkonzentrationen im Ablauf der Kläranlagen etwa eine Zehnerpotenz über den Konzentrationen bei Trockenwetter und etwa eine Zehnerpotenz unterhalb der in den Mischwasserentlastungen gemessenen Konzentrationen. Durch die erhöhte Keimbelastung im Kläranlagenablauf bei Regenwetter und dem geringen Konzentrationsunterschied zu den Mischwasserentlastungen kann eine höhere Beschickung der Kläranlagen im Regenwetterfall (integraler Ansatz) nicht wesentlich zu einer Reduzierung der Keimfrachten aus Abwasseranlagen beitragen.

Die Mischwassereinleitungen aus Regenüberlaufbecken tragen zusätzlich zu einer massiven hygienischen Gewässerbelastung bei. In den untersuchten Gewässern konnten bei Regenwetter zu keiner Zeit die Anforderungen nach EG-Badegewässerrichtlinie (2006/7/EG) erfüllt werden. Dies gilt auch für Gewässerstellen, die noch anthropogen unbelastet waren.

Die hygienischen Gewässerbelastungen sind nicht ausschließlich punktuellen Eintragspfaden zuzuordnen, sondern sie sind vielmehr eine Summe der komplexen Einflüsse natürlicher und anthropogener Komponenten. Der Einfluss der unterschiedlichen Eintragspfade variiert in Abhängigkeit des Gewässerabflusses, aber auch in Abhängigkeit der Flächenanteile Wald, Landwirtschaft, Siedlungsbereiche, Anzahl und Belastung der Nebengewässer, bewirtschaftetes Rückhaltevolumen etc.

Um eine kosteneffiziente Optimierung der Immissionssituation zu erreichen, müssen sorgfältige Bestandsaufnahmen durchgeführt werden. Dabei darf der Schwerpunkt nicht ausschließlich auf der Siedlungsentwässerung liegen, sondern muss diffuse Einträge mit einbeziehen.

\section{Korrespondenz:}

Dr.-Ing. Juliane Gasse

Institut für Siedlungswasserbau, Wassergüte- und Abfallwirtschaft der Universität Stuttgart,

Arbeitsbereich Abwassertechnik

Bandtäle 2

D-70569 Stuttgart-Büsnau

Tel: + 49 (0)71168565410

E-Mail: Juliane.Gasse@iswa.uni-stuttgart.de 